\title{
Analyse der Persönlichkeit
}

Ursprünglich für die Psychologie entwickelt hat sich der Einsatz von Persönlichkeitsanalysetools in den letzten Jahren immer stärker auch im allgemeinen Gebrauch durchgesetzt. Inzwischen beschränkt sich der Einsatz derartiger Verfahren aber schon lange nicht mehr nur auf kleine Selbstanalysen aus Zeitschriften bzw. entsprechender Ratgeberliteratur zur Persönlichkeitsentwicklung, sondern hat vor allem im Unternehmenseinsatz datenschutzrechtliche Bedeutung erlangt. Dabei wird auch dort die zunächst vorwiegende Nutzung im Bereich von Bewerbungsverfahren (sogenannten Assessment-Centern) inzwischen ausgeweitet, sodass sich Mitarbeiter und Mitarbeiterinnen immer häufiger selbst in einem bereits bestehenden Beschäftigungsverhältnis mit der Notwendigkeit der Teilnahme an solchen Persönlichkeitsanalysen konfrontiert sehen. Gerade die größeren und zumeist international agierenden Unternehmen sehen darin die Möglichkeit, die Eigenschaften ihrer Mitarbeiterinnen und Mitarbeiter in Führungspositionen - aber auch in Projekten und kleineren Teameinsätzen - besser einschätzen zu können und deren Einsatz von den Ergebnissen derartiger Tests abhängig zu machen. Dabei liegt das Problem nicht allein darin, dass die wissenschaftliche Fundiertheit solcher Persönlichkeitsanalyseverfahren zumeist gar nicht festzustellen ist. Datenschutzrechtlich betrachtet liegt die Gefahr für die informationelle Selbstbestimmung vor allem darin, dass mit der Beantwortung der gestellten Fragen, deren Auswertung und eventuell auch noch vorgenommener Fremdeinschätzungen (beispielsweise durch Interviews) eine Vielzahl an personenbezogenen Daten gesammelt werden, die je nach Reichweite sogar besondere Arten personenbezogener Daten umfassen können. Die Verarbeitung solcher Daten wird aber oftmals gar nicht - oder zumindest nur wenig kritisch - hinterfragt. Vielmehr wird zumeist die Einwilligung des Betroffenen als ausreichend erachtet, um dann sämtliche Verarbeitungsvorgänge darauf zu stützen. Das allein reicht aber nicht aus, um die datenschutzkonforme Umsetzung im Unternehmen zu gewährleisten. Ein Unternehmen, welches solche Persönlichkeitsanalysetools einsetzen möchte, sieht sich demzufolge gut beraten, wenn es zunächst die datenschutzrechtlichen Fragen klärt.

Einige dieser datenschutzrechtlichen Schwierigkeiten werden daher in diesem Heft aufgegriffen. Hierzu wird zunächst im Beitrag von Christoph Bausewein allgemein dargestellt, worauf Unternehmen bei der Auswahl und dem Einsatz derartiger Verfahren achten müssen sowie welche datenschutzrechtlichen Maßgaben zu beachten sind. Jens Eckhardt und Rudi Kramer beschäftigen sich sodann verstärkt mit der datenschutzrechtlichen Schwierigkeit der Einbindung externer Dienstleister mithilfe der Auftragsdatenverarbeitung (ADV), bevor Christian Kuß sich in seinem Aufsatz mit dem gerade auch in Rahmen des Einsatzes von Persönlichkeitsanalysetools relevanten datenschutzrechtlichen Problem der Strukturierung eines Unternehmens in einer Matrixorganisation beschäftigt.

Außerdem soll die aktuelle Entwicklung der europäischen Datenschutz-Grundverordnung (DS-GVO) ebenfalls nicht unbeachtet bleiben, hat sich hierzu doch in den letzten Wochen einiges getan. Im Beitrag von Benedikt Buchner wird deswegen ein erster Ausblick auf die zu erwartenden Grundsätze und die Rechtmäßigkeit einer Datenverarbeitung bei Umsetzung der europäischen Datenschutz-Grundverordnung gegeben und deren Harmonisierungskraft und ihre Auswirkung auf nationales Datenschutzrecht diskutiert.

\section{Britta Alexandra Mester}

